Alto, California, USA; ${ }^{3}$ Depatment Rheumatology, Leiden University Medical Centre, Leiden, The Netherlands

10.1136/ard.2010.148965.8

Objective To determine the effects of the B cell depleting drug rituximab on gene expression in synovial tissue from patients with refractory rheumatoid arthritis (RA) who have been on rituximab for 2 years.

Methods Synovial tissue biopsies were collected from 19 patients with refractory RA at baseline, and 3 months after the first infusion of rituximab. 117 genes of interest were selected for analysis, including immune cell genes and fibrosis genes. Analysis was carried out using microfluidic real-time q-PCR and CT values were then obtained using the fluidigm gene expression data analysis software (version 2.1.1 Fluidigm).

Results B cell depletion was evident in both responders and non-responders of rituximab between baseline and 3 months as assessed by CD20 gene expression. An unsupervised hierarchical cluster analysis revealed the presence of two groups of RA patients. One with high inflammation at baseline and one with low inflammation at baseline. The high inflammation group had significantly higher Disease Activity Score using 28 joint counts (DAS28), higher $C$ reactive protein levels, higher erythrocyte sedimentation rate and a greater change in DAS28 over 3 months.

Conclusion These results show the existence of two distinct RA patient subgroups, where high inflammation tissue is associated with more severe disease and a better response to rituximab.

\section{A38 CHANGES IN GENE EXPRESSION IN SYNOVIAL TISSUE FROM REFRACTORY RHEUMATOID ARTHRITIS PATIENTS TREATED WITH RITUXIMAB}

Vanessa Hogan, ${ }^{1}$ Cecile Holweg, ${ }^{2}$ Sarah Kummerfeld, ${ }^{2}$ Y K Onno Teng, ${ }^{3}$ Michael Townsend, ${ }^{2}$ Jacob M van Laar' ${ }^{1}$ Musculoskeletal Research Group, Institute of Cellular Medicine, Newcastle University, Newcastle Upon Tyne, UK; ${ }^{2}$ Genentech, Palo 